\title{
A test for checking earthquake aperiodicity estimates from small samples
}

\author{
M. Mucciarelli \\ Dept. of Structures, Geotechnics and Engineering Geology, Univ. of Basilicata, Potenza, Italy
}

Received: 28 February 2007 - Revised: 31 May 2007 - Accepted: 31 May 2007 - Published: 13 June 2007

\begin{abstract}
In recent years, many new models for earthquake recurrence were proposed. Some are focusing on the clustering properties on a small time scale, while others try to model the long term behavior of large mainshocks. To this last purpose, there is a growing interest for models that take into account the aperiodicity aiming to a time-dependent hazard estimate. It is well known that a limited number of inter-event times (IETs) may lead to biased values of the distribution parameters. To overcome this problem different solutions were proposed. This paper focuses on two of them: Monte Carlo simulation of the process and aperiodicity estimated via a statistical proxy. The topics discussed are: 1) how many IETs are needed for a correct estimate, 2) to which extent a Poisson distribution is equally able to describe the process, 3 ) the influence of errors associated to paleoseismological IETs, and 4) the goodness of the success ratio from simulations. A simple test is proposed to discriminate real aperiodicity from apparent aperiodicity coming from undersampling.
\end{abstract}

\section{Introduction}

The standard Poisson process requires only one parameter to model the occurrence of earthquakes, that is the mean recurrence time $(\mu)$. The standard deviation $(\sigma)$ is assumed to be equal to the mean. The ratio $\alpha=\sigma / \mu$ (coefficient of variation or aperiodicity) is taken as an indication of statistical properties of the relevant time series and used in renewal models for time-dependent hazard analysis. When $\alpha>1$, the time series exhibits clustering properties. Values $<1$ indicate the possible presence of periodicity, with increasing probability for decreasing $\alpha$. The Log-normal, Weibull and Brownian Passage Time (BPT) models require to estimate from sample data both $\mu$ and $\alpha$. Performing a bootstrap numer-

Correspondence to: M. Mucciarelli

(marco.mucciarelli@unibas.it) ical simulation for 100 realizations of a BPT process with $\mu=1$ and $\alpha=0.5$, Ellsworth et al. (1999) showed that $\alpha$ is underestimated when the number of events in the catalogue is small $(<10)$. Parsons (2005) implemented a more sophisticated Monte Carlo simulation of a BPT, trying to simulate observed sequences with a range of mean IET and aperiodicity. Events were repeatedly drawn at random from each distribution, attempting to match the observed event windows. The two sequences studied occurred both on the San Andreas Fault: 14 event at Wrigthwood (Fumal et al., 2002) and 10 events at Pallet Creek (Sieh et al., 1989). Each distribution was sampled 5 million times, with a success ratio equal to $5.410^{-6}$ for Wrigthwood and $2.810^{-6}$ for Pallet Creek.

The long paleoseismological records obtained in California can be hardly matched in other countries. The increasing number of studies on active seismogenic sources prompted researchers to find new ways of including these data in seismic hazard estimates. An example is coming from Italy: in recent years several studies on seismogenic sources allowed for the creation of an on-line data base (DISS Working Group, 2006). On the other hand, attempts were made to include paleoseismological data in seismic hazard estimates: the most recent example is given by Pace et al. (2006). In the area they studied (Central Italy) the longest record available is for the Fucino fault, with 5 events in about 8000 years (Galadini and Galli, 1999). Pace et al. (2006) recognized that the number of IETs was not sufficient for an unbiased estimate of $\alpha$, so they decided to use a statistical proxy of aperiodicity: for each fault they derived $\mu$ and $\sigma$ from the distribution of mean recurrence times obtained with different techniques from the same dataset. The distribution of $\sigma$ versus $\mu$ and the histogram of $\alpha$ are reported in Fig. 1. It can be noted that the aperiodicity is concentrated around few values. If compared with the values obtained by Parsons (2006) it is also very small, leading to suspect a strong bias. At this point a series of simulations were carried out to try to answer some questions:

Published by Copernicus Publications on behalf of the European Geosciences Union. 

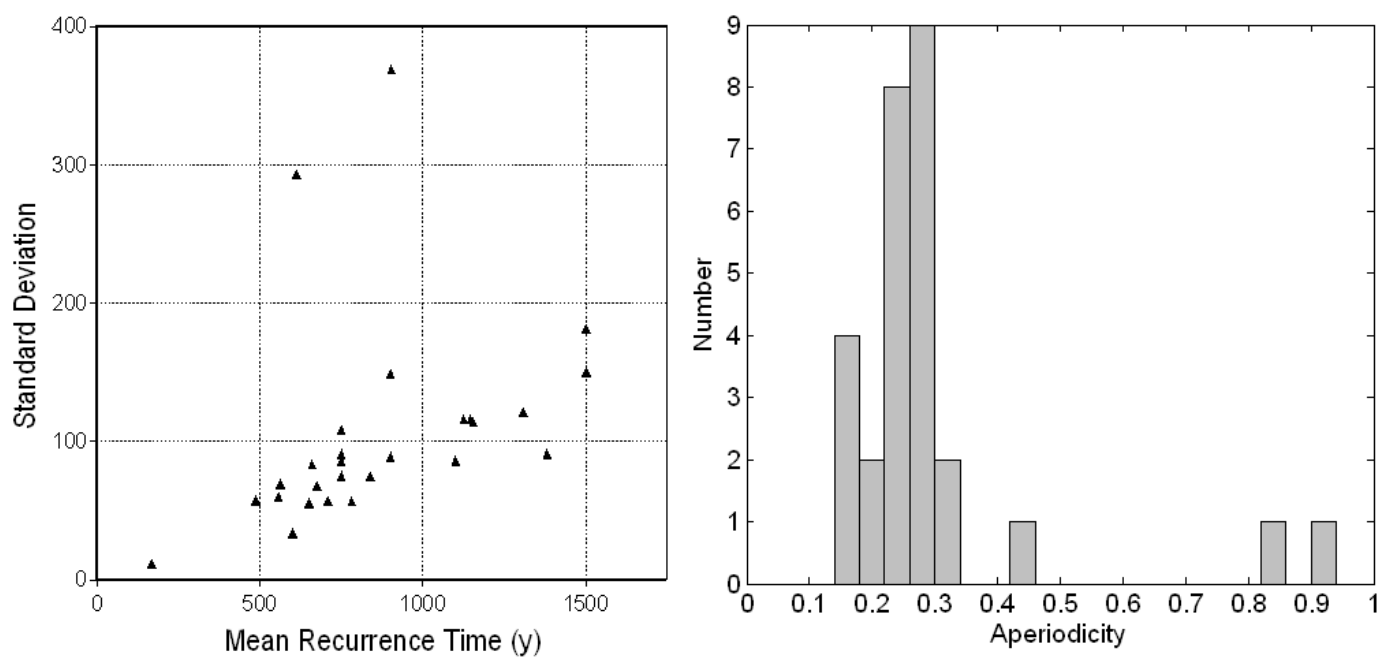

Fig. 1. The distribution of $\sigma$ versus $\mu$ (left) and the histogram of $\alpha$ (right) for 28 seismogenic sources in Central Italy, according with Pace et al. (2006).

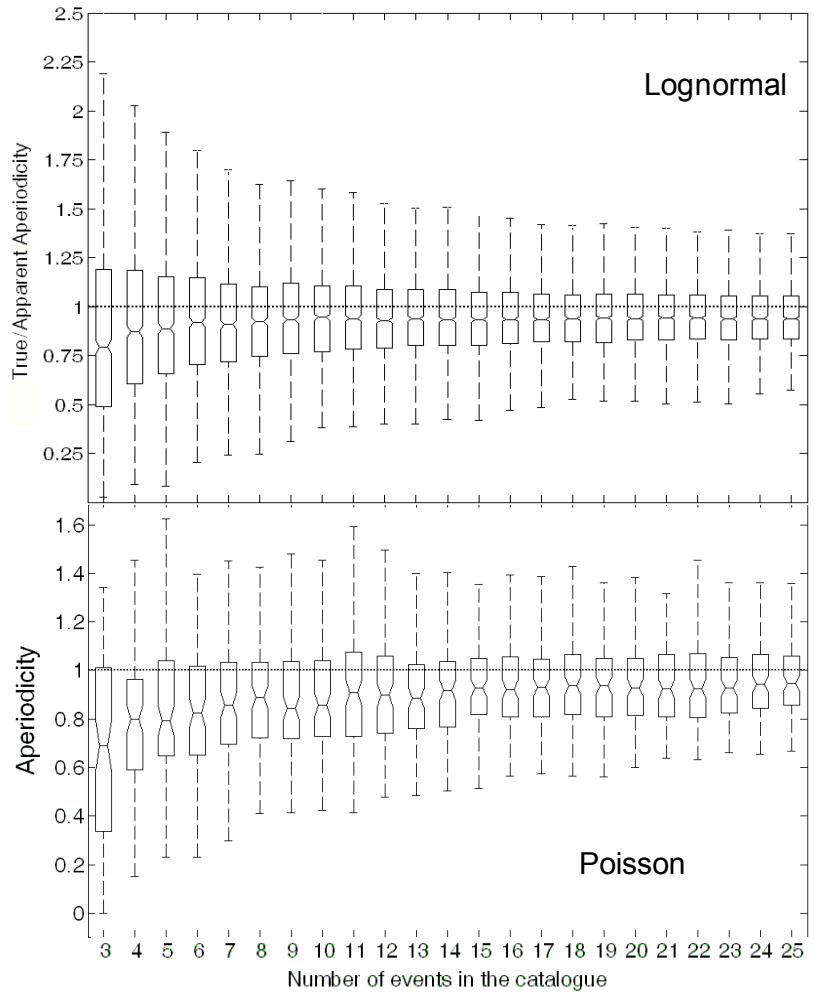

Fig. 2. Numerical estimate of the underestimate of $\alpha$ with a bootstrap procedure on 100 catalogues composed of 100 events each. For the two-parameters distribution the seed values were $\mu=1$ and $\sigma$ uniformly distributed between 0.95 and 1.05 . The investigated parameter is the ratio between the true $\alpha$ and the one retrieved from the synthetic catalogues. For the Poisson case, the statistic is $\alpha$ retrieved from data, or apparent aperiodicity.
- How many IETs are needed for a correct estimate?

- To which extent a Poisson distribution is equally able to describe the observed processes?

- Which is the influence of errors associated to paleoseismological IETs?

- Which is he reliability of the success ratios obtained from simulations?

\section{Preliminary modeling and simulations}

As mentioned above, Ellsworth et al. (1999) showed that for a BPT process the estimate of $\alpha$ is biased when the number of event is $<10$. In the following of this paper the ability of a Poisson process to reproduce the observed data will be discussed, so it was interesting to compare the bias in aperiodicity estimate between a distribution with two free parameters and one with a single parameter.

The first step was the generation of two sets of 100 catalogues composed of 100 events each. For the two-parameter distribution (Log-normal or BPT), the values were $\mu=1$ and $\sigma$ uniformly distributed between 0.95 and 1.05 . In this case the investigated parameter was the ratio between the true $\alpha$ (that is the one used as seed of each simulation) and the one retrieved from the synthetic catalogues. For the Poisson case, the statistic was simply $\alpha$ retrieved from data, or apparent aperiodicity. The desired parameters were estimated for each catalogue using a bootstrap procedure, with the number of events varying from 3 to 100. The results are shown in Fig. 2 for the first 25 events (after that value the asymptotic behavior continues smoothly and converges to unity for more than 50 events). The Poisson process shows a slightly larger underestimate of the true aperiodicity. It is important to note 


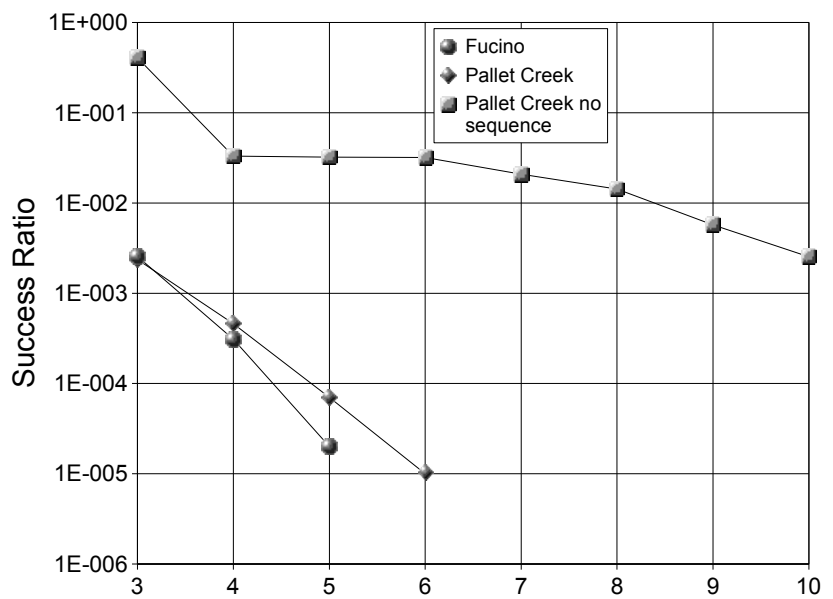

Fig. 3. Bootstrap procedure simulating 100.000 realizations of two Poisson processes having $\mu$ estimated from the Fucino data ( $\mu=1498)$ and from the most recent events of the Pallet Creek sequence $(\mu=120)$. For the Pallet Creek data set the comparison is also with or without the request for exact matching of the sequence (less restrictive, exact Poissonian condition; see text for details).

that here it is not included the effect on the estimate caused by the "seismic drought" (Davis et al., 1989): this issue will be discussed later. Another important point for further discussions is that for a number of events equal to the longer sequence available in Italy ( 5 earthquakes on the Fucino Fault), the apparent aperiodicity for a true Poisson process is 0.8 , with $50 \%$ of the bootstrap values in the interval $0.70 \div 1.05$. According with Galadini and Galli, the last 5 events on the Fucino fault occurred in 1915 AD, 508-618 AD, 1700-1300 BC, 3944-3516 BC, 5979-5576 BC. There are two previous events, but with a large overlapping time window that prevent their use in this analysis. Taking the midpoint of the intervals, the estimate of the parameters is $\mu=1923.1 \pm 389.6$ years. The aseismic period since the last event is $91 \mathrm{y}$ (less than $5 \%$ of $\mu$ ) so according to Davis et al. (1999) the "seismic drought" effect is negligible. The aperiodicity estimates gives $\alpha=0.2$ : if compared with Fig. 2, it may seem unlikely that this distribution is Poissonian. More insights on the statistics of the Fucino sequence may come from simulations similar to those proposed by Parsons (2005). Figure 3 reports the results of a bootstrap procedure simulating 100.000 realizations of two Poisson processes having $\mu$ estimated from the Fucino data and from the most recent events of the Pallet Creek sequence. With a limited number of events, a Poisson process is able to reproduce the sequences with a success ratio much higher than the one obtained for the full sequence by Parson $(2005)$ with a BPT process $\left(\approx 10^{-6}\right)$. The simulation was not carried out for the whole Pallet Creek sequence, but the extrapolation of the negative exponential trend in Fig. 3 gives an estimated success ratio $\approx 10^{-8}$.

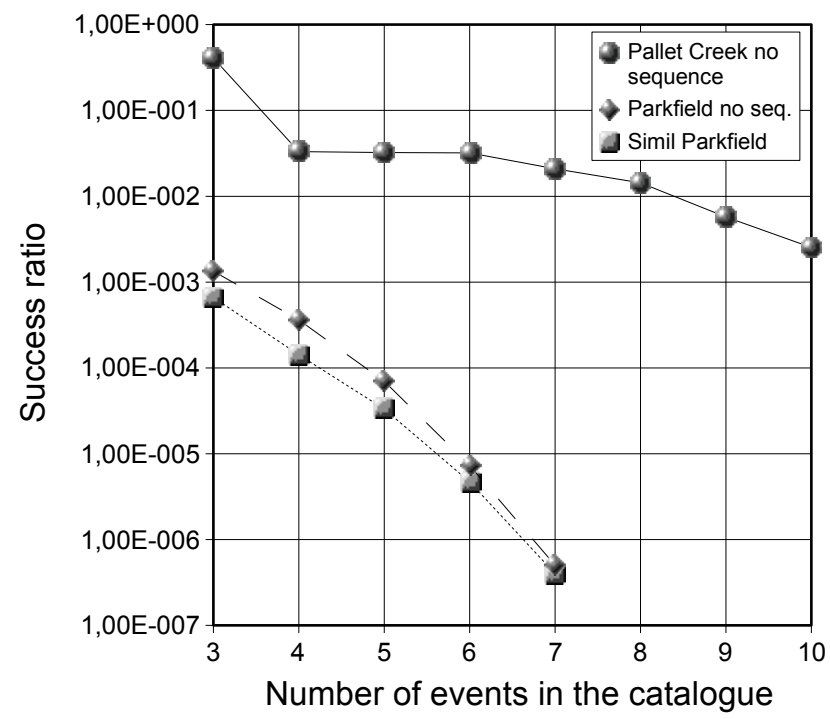

Fig. 4. Bootstrap simulation of Pallet Creek and Parkfield sequences, plus a sequence generated with a Poisson process with the same $\mu$ of Parkfield. The difference in success rate is due to the large errors in the paleoseismological datations, which are absent in the historical sequence of Parkfield.

The simulations were carried out imposing that the sequence of IETs should match exactly the real one. This makes more sense if one aim to simulate a renewal process, where the memory of the system has to be taken into account. But for a Poisson process, the exact temporal sequence of the IETs is not an issue: it is sufficient that each one of the simulated recurrence times falls within the boundary of one (and only one) of the intervals from the real data. This new condition on the simulations greatly improves the success ratio. Figure 3 shows what happens to the Pallet Creek data set: the whole sequence can be simulated by a single-parameter Poisson process with a success ratio $10^{-3}$ times greater than the one obtained by Parsons (2006) and $10^{-5}$ times greater than the one previously estimated. Thus the conditions imposed on the simulation regarding the memory of the system have a very strong influence on the result.

Another factor that has to be investigated is the error associated to the paleoseismological data. The influence of the datation errors can be evaluated using a sequence composed of historical data only. The Parkfield sequence provides a very good example. Bakun and Lindh (1985) suggest that characteristic $\mathrm{M}=6$ earthquakes occurred in 1857, 1881, 1901, 1922, 1934, and 1966. Then in 2004 the 7th event occurred, spurring a debate on the characteristic earthquake model itself (Jackson and Kagan, 2006). 10 million simulations were run, using $\mu=24.5$ and comparing against the real Parkfield sequence and against a Poisson sequence randomly generated using the same $\mu$. Also in this case the less restrictive rule on sequences was applied. The results are shown in Fig. 4: the two simulations give very similar results, but with 


\section{Fucino sequence}

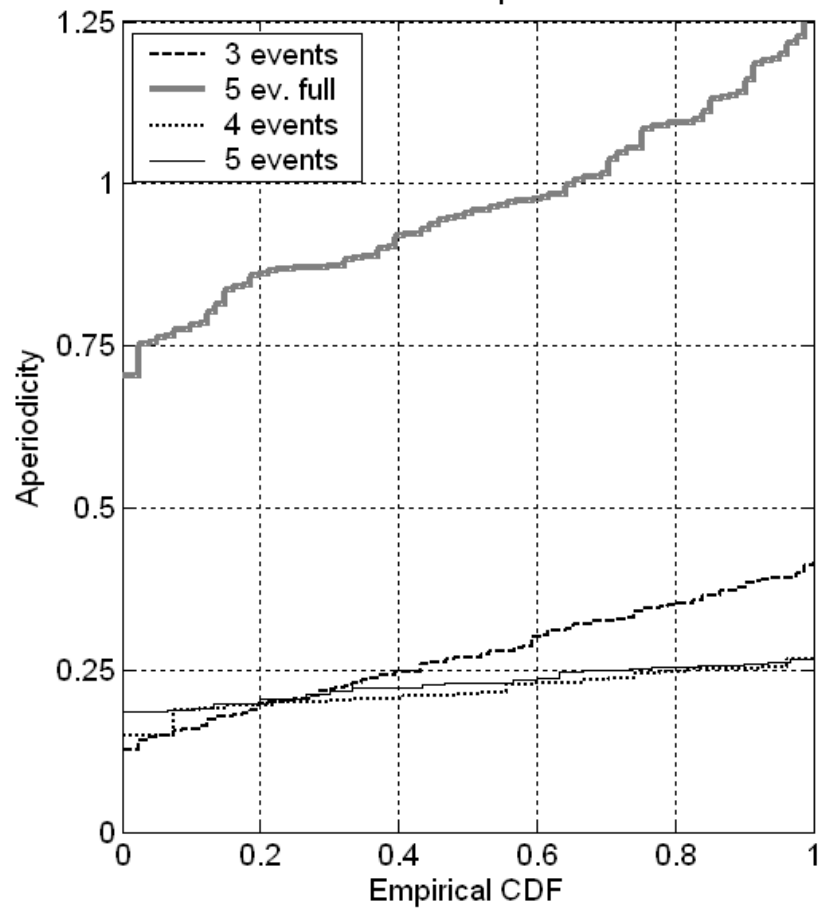

Fig. 5. ECDFs of aperiodicity from the first $n$ IETs and from the whole sequences that match the Fucino data set (Poisson process, $\mu=1923$ from real data, no restriction on exact sequence).

a success ratio much smaller than the Pallet Creek one. The drop in the success ratio is in the range $10^{2-} 10^{3}$. It is interesting to note that the values obtained for Parkfield are similar to those obtained for Fucino and Pallet Creek sequences in Fig. 3. This means that reducing the errors on paleoseismological data decreased the success ratio of the same amount it is increased by lifting the restrictive condition on the exact match of the sequence.

\section{Testing aperiodicity from small samples}

The test proposed for checking the earthquake aperiodicity from short catalogues is based on the following assumptions, derived from the insight gained in the previous chapter:

1. When small samples are considered, it is better to start with 1-parameter simulation rather than 2-parameters simulations. If the real sequence has a significant aperiodicity, it can be observed and proved against the null hypothesis of Poissonian behavior.

2. Dealing with Poisson processes, the restriction on the exact match between observed and simulated sequences can be lifted. Instead, it will be sufficient that each one of the simulated recurrence times must fall within the

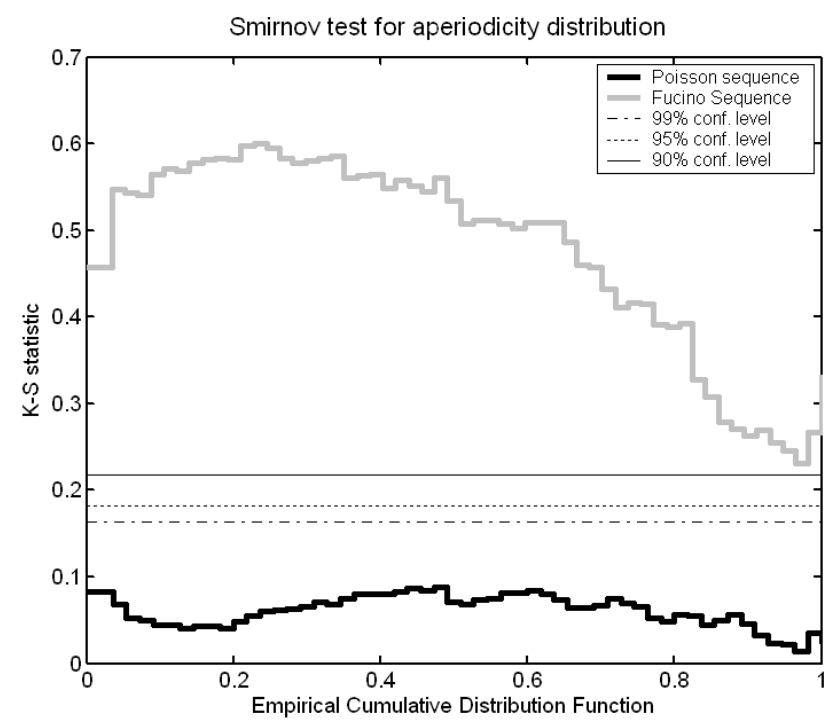

Fig. 6. K-S statistics for the full Fucino sequence $(n=4)$ and for a Poisson process generated with $\mu=1923$ y and an error similar to the paleoseismological data. The confidence limits refer to the rejection of equality hypothesis in the Smirnov test.

boundary of one (and only one) of the intervals from the real data.

3. The errors on the datation of pre-historic events greatly affect the absolute value of success ratio. Their effect on the trend of success ratio versus the number of events in the catalogue is less important. Thus, the success ratio alone is a poor estimate of the goodness-of-fit of our simulations, given its large variability. Instead, the simulations that match real sequences can be compared against single realization of a Poisson process having the same $\mu$.

If the above is true, then a simple procedure is implemented in four steps:

1. Generate a set of two-parameters synthetic sequences $(S)$ longer than the real one $(R)$. Repeated test showed that 50 events are sufficient. Then, check for each $S$ sequence if first $n$ IETs match the $n$ IETs of the $R$ sequence;

2. For the $S$ sequences that pass the above check, estimate the aperiodicity from the first $n$ IETs $\left(\alpha_{n}\right)$ and then from the whole sequence $\left(\alpha_{50}\right)$.

3. Calculate the Kolmogorov-Smirnov statistic (K-S $S$ ), that is the absolute value of the maximum difference between the two empirical cumulative distribution functions (ECDFs) of $\alpha_{n}$ and $\alpha_{50}$;

4. generate a Poisson process with the same $\mu$ of the $R$ sequence, then repeat step 1 and 2 to obtain the statistic K-S $R$. 

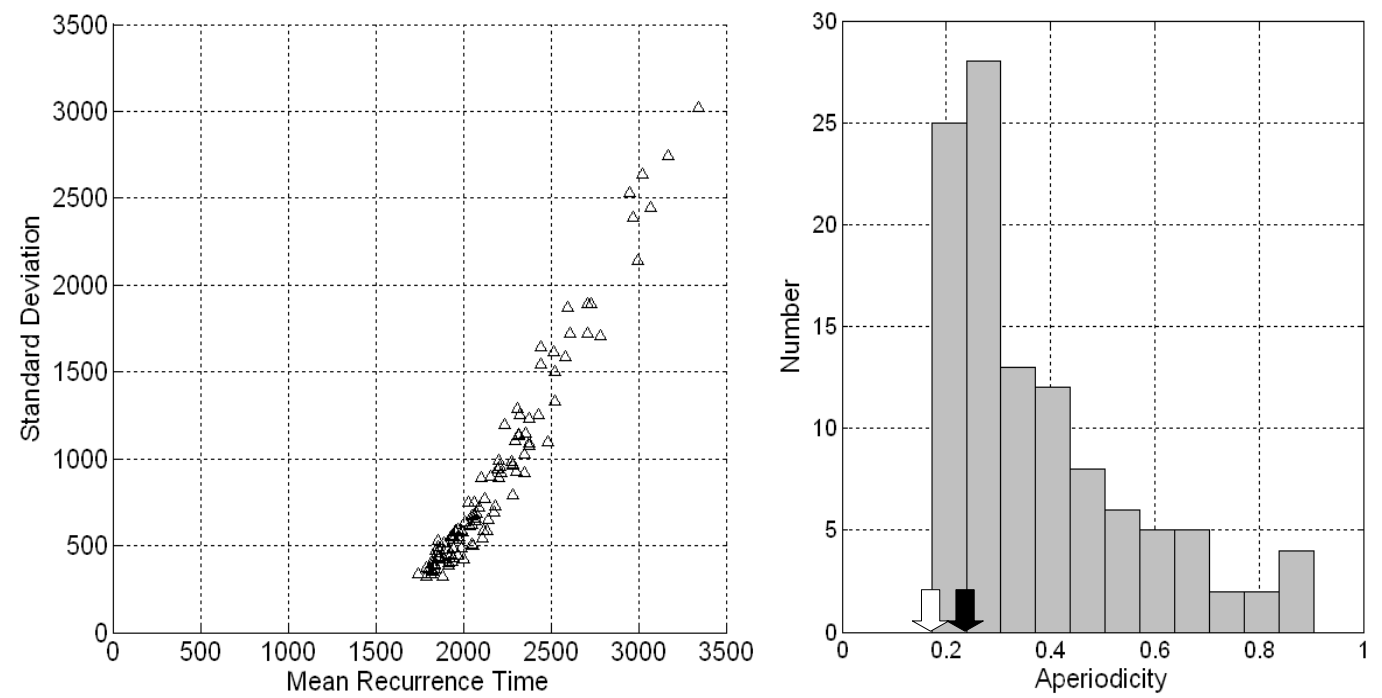

Fig. 7. The distribution of $\sigma$ versus $\mu$ and the histogram of $\alpha$ obtained for the Fucino sequence with 100.000 trials. The black arrow marks the $\alpha$ obtained for the Fucino fault by Pace et al. (2006), while the white one is the $\alpha$ estimated directly from the data given by Galadini and Galli (1999).

5. perform a Smirnov test on the K-S $S$ and K-S $R$ statistics. The second one should always pass the test at any significativity level, and act as a control group. If the first set does not pass the test, this means that we may suppose that the real IETs distribution is not Poissonian.

Let us examine the practical case of the Fucino sequence. To check if the proposed procedure is reliable also for smaller sequences, the first two steps are performed for $n=2,3,4$. The results is shown in Fig. 5. The aperiodicity estimated from the whole sequences is much higher, and the mean value is close to 1 , as expected for a Poisson process. The aperiodicity estimated from the first $n$ IETs yields a much lower value, and the ECDFs are similar for $n=2,3,4$. Figure 6 shows the K-S statistics for the full Fucino sequence $(n=4)$ and for a Poisson process generated with $\mu=1923$ years and an error similar to the paleoseismological data. While the Kolmogorov-Smirnov test verify if an empirical CDF is different from a theoretical one, the Smirnov test checks if the hypothesis that two ECDFs are coming from the same population can be rejected. In both cases, the inequality of distributions is proved if the maximum value of K-S statistics exceeds a given confidence level. In this case there are no doubt about rejecting the equality of the ECDFs for the real case. The difference between the ECDF of the aperiodicity estimated for the first 4 IETs and for the full sequence is always greater than the test limits. A collateral advantage of the proposed procedure is that at this point we can use the distribution of aperiodicity estimated from the first $n$ synthetics IETs. Figure 7 shows the distribution of $\sigma$ versus $\mu$ and the histogram of $\alpha$ obtained for the Fucino sequence with 100000 trials. The success rate is about $1.10^{-3}$. The black arrow marks the $\alpha$ obtained for the Fucino fault by Pace et al. (2006), while the white one is the $\alpha$ estimated directly from the data given by Galadini and Galli (1999). Both appears to a lower bound of the distribution.

\section{Discussion and conclusions}

The simulation of synthetic sequences of earthquakes on a single fault (Parsons, 2005) proved to be an effective tool for the understanding the time distribution of events. However, the success ratio alone is a poor estimate of the goodnessof-fit of our simulations, given its large variability due to the width of errors on paleoseismological datation and more or less restrictive hypothesis on exact match of real data. A simple technique is proposed to evaluate if the aperiodicity estimated from short catalogues is real. The test was checked on the longest sequence of earthquakes associated to a single fault in Italy, the Fucino sequence. Even for a reduced number of events, it is possible to reject the hypotehsis that the data are coming from a pure Poisson process.

Acknowledgements. I am indebted with E. Di Nardo at the Mathematical Dept. of Basilicata University for checking the statistical hypothesis. This work started from discussions had during the workshop "EARTHQUAKE AND SHAKING PROBABILITIES", Erice (Italy), October 2006: thanks to the organizers for their efforts in making it possible. Many thanks to M. R. Gallipoli and P. Galli for their help and discussions. Thanks to D. Albarello and another anonymous reviewer for improving the manuscript. Financial support was provided by project DPC-INGV-S2. 
Edited by: M. Contadakis

Reviewed by: D. Albarello and two other referees

\section{References}

Bakun, W. H. and Lindh, A. G.: The Parkfield, California, earthquake prediction experiment, Science, 229, 619-624, 1985.

Davis, P. M., Jackson, D. D., and Kagan, Y. Y.: The longer it has been since the last earthquake, the longer expected time till the next?: Bull. Seism. Soc. Am., 79, 1439-1456, 1989.

DISS Working Group: Database of Individual Seismogenic Sources (DISS), Version 3.0.2: A compilation of potential sources for earthquakes larger than M 5.5 in Italy and surrounding areas, www.ingv.it/DISS/, 2006.

Fumal, T. E., Weldon II, R. J., Biasi, G. P., Daeson, T. E., Seitz, G. G., Frost, W. T., and Schwartz, D. P.: Evidence for large earthquakes on the San Andreas fault at the Wrightwood, California, Paleoseismic site: A.D. 500 to present, Bull. Seismol. Soc. Am., 92, 2726-2760, 2002.
Ellsworth, W. L., Matthews, M. V., Nadeau, R. M., Nishenko, S. P., Reasenberg, P. A., and Simpson, R. W.: A PhysicallyBased Earthquake Recurrence Model for Estimation of LongTerm Earthquake Probabilities, U.S.G.S. Open File 522, 22 pp., 1999.

Galadini, F. and Galli, P.: The Holocene paleoearthquakes on the 1915 Avezzano earthquake faults (central Italy); implications for active tectonics in the central Apennines, Tectonophysics, 308, 143-170, 1999.

Jackson, D. D. and Kagan, Y. Y.: The 2004 Parkfield Earthquake, the 1985 Prediction, and Characteristic Earthquakes: Lessons for the Future, Bull. Seism. Soc. Am., 96, S397-S409, 2006.

Pace, B., Peruzza, L., Lavecchia, G., and Boncio, P.: Layered Seismogenic Source Model and Probabilistic Seismic-Hazard Analyses in Central Italy, Bull. Seism. Soc. Am., 96, 107-132, 2006.

Parsons, T.: Significance of stress transfer in time-dependent earthquake probability calculations, J. Geophys. Res., 110, B05S02, doi:10.1029/2004JB003190, 2005.

Sieh, K., Stuiver, M., and Brillinger, D.: A more precise chronology of earthquakes produced by the San Andreas fault in southern California, J. Geophys. Res., 94, 603-623, 1989. 